\title{
Temperature Behavior Visualization on Rubber Material Involving Phase Change Simulation
}

\author{
Roziha Darwis ${ }^{2, *}$, Norma Alias ${ }^{1}$, Nazeeruddin Yaacob ${ }^{1}$, Mohamed Othman ${ }^{3}$, Nurashikin Abdullah ${ }^{2}$, Teh \\ Yuan Ying ${ }^{2}$ \\ ${ }^{1}$ Mathematics Department, Ibnu Sina Institute, University Teknologi Malaysia, Skudai, Johor, Malaysia. \\ ${ }^{2}$ Mathematics Department, Faculty of Science, University Teknologi Malaysia, Skudai, Johor, Malaysia. \\ ${ }^{3}$ Department of Communication Technology and Networking, Faculty of Computer Science and Information Technology, \\ Universiti Putra Malaysia, Serdang, Selangor, Malaysia. \\ *Author to whom correspondence should be addressed; E-mail: roziha.darwis@gmail.com
}

Received: 31 October 2008, Revised: 17 February 2009

Online Publication: 27 May 2009

http://dx.doi.org/10.11113/mjfas.v5n1.287

\section{ABSTRACT}

Material engineers are excited with the design of a new rubber product through the development of a new composite of the rubber product. Our research contributes in developing the mathematical simulation based on Gauss-Seidel RedBlack and Gauss-Seidel method to solve the temperature behavior of the rubber elasticity, strength, entropy and classical experiments through reference publications and stimulating rubber physics research elsewhere. The temperature behavior leads to the partial differential equation of heat transfer problems involving phase change simulation. The prototype of the algorithm implemented on Linux operating systems using $C$ language.

| Rubber | Temperature Behavior | Heat Transfer | Phase Change Simulation | Parabolic Equation | Latex |

\section{Introduction}

Heat transfer process occurs due to the polymer flow as convection. The motion of fluid transfers an energy along its flow path and thus convects heat during mould filling (Davis et Al., 2003). To predict the temperature behavior on rubber material involving phase change processes, this prediction solving by the mathematical simulation. A mathematical model for the prediction of temperature profiles and heat transfer rates during the blow moulding process (Edwards et Al., 1981). This paper focuses on the research to study the influence of operating conditions on cooling time. The experimental attention to be focused on to using a chilled mould and gas circulation to give enhanced cooling rates. Analytical data obtained on a small laboratory at Lembaga Getah Malaysia as an exact solution, and limited to testing on an industrial production line for the manufacturing of large barrels have been confirmed the validity of theoretical approach.

Deqian (1986) has presented a fundamental method for the heat transfer calculation in polymer processing. Calculation of the heat transfer with viscous heat generation for the drag flow of rubber melts between two coaxial cylinders by using the finite difference method is carried out. The inner cylinder is rotating at a steady 
angular velocity, but the outside of a cylinder is fixed. As the rubber melting passes through the extrude rubber, it will undergo intensive shear friction and much more viscous heat will be generated. The temperature distribution along the rotating downstream length was obtained, and the approximate temperature gradients on the cylindersurfaces were calculated. The calculated result has been shown that average melt temperature on the section of the two cylinders gradually rises along the rotating downstream length, but the temperature gradient reduced extremely slowly. The fully developed of temperature profile has then been constructed. The numerical methods under considerations are some finite difference methods. These numerical methods are straightforward built on Linux platforms.

A modeling of curing reaction of a maleic polyester resin during the compression moulding process has been presented by Azaar et al., 1992. The objectives of the studies are to predict both the temperature profiles and state of cure profiles developed within the sample at any time, as well as the temperature-time histories at the midplane for the thin sheet resin and to investigate the effect of the rate of stirring the oil which heats the mould and resin. The temperature-time histories at the middle of thin sheets of resin obtained from the experiments and calculations were compared in order to test the validity of a model.

\section{Material Properties}

Rubber is an elastic hydrocarbon polymer that naturally occurred as a milky colloidal suspension, or latex, in the sap of some plants and it can be synthesized. It is found in about 2000 different plant species all over the world, the only rubber-producing plant of commercial interest is the para rubber tree or the scientific name is Hevea brasiliensis (Bengtsson and Stenberg, 1996). Other plants that contain rubber are Guayule, a shrub growing in Mexico and the southern part of the USA, and the Balata tree. The rubber from Guayule is cis-1, 4polyisoprene, while the rubber from the Balata tree is trans-1,4-polyisoprene.

Rubber exhibits with unique physical and chemical properties. Rubber's stress-strain behavior exhibits the Mullins to be accomplished, the Payne effect and is often modeled as hyper elastic. The synthetic rubber can be made as a polymer of isoprene or various other monomers. The material properties of natural rubber will make it an elastomer and a thermoplastic. However, it should be noted that as the vulcanization of rubber it would turn into a thermoset.

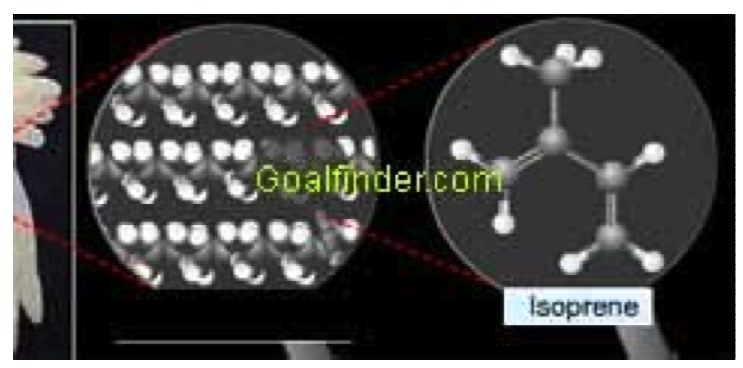

Figure 1: Chemical-rubber-poly-cis-isoprene.

Latex contains rubber hydrocarbon and a large amount of non-rubber constituents, which present relatively small amounts. Many of these non-rubber constituents are dissolved in the aqueous serum of latex, while others might be absorbed on the surface of the latex itself. Some others might exist as rubber particles suspended in the latex. As a natural product, the composition of freshly tapped latex varies between wide limits, with typical composition such as total solid content, dry rubber content, protein substances, neutral lipids as well as phospholipids, ash, carbohydrates and inositols, nitrogen compounds and water. Due to commercial purposes, 
accelerators, antioxidants and adulterants might be added to freshly tapped latex to enhance the quality of the latex.

The components of chemical are existed in fresh latex lipid. Lipid is a water-soluble substance and maybe divided two portions which are neutral lipid and polar lipid. Phospholipids are polar lipids that can be found in freshly tapped Hevea latex. Neutral lipids found in fresh latex contain substances such as carotene pigments and esters, triglycerides, tocotrienols, sterols and mixture of fatty acids, fatty alcohols, di- and monoglycerides.

Latex might undergo some process to change to rubber. This purpose will change the composition of the lipids present. Portion of lipid that might have retained in the processed latex is usually the neutral lipids, while polar lipids will be degraded. The degradation of the polar lipids portion might result in a significant increase of the fatty acids in the processed latex.

In the factory, there are various processes to convert field latex into dry rubber. This process is always achieved by a coagulation process. The type of coagulation has a special influence on the rubber properties when worked industrially and on a finished rubber article (Bengtsson and Stenberg, 1996). The different types of coagulation are auto-coagulation, acid coagulation and steam coagulation. After a coagulation process, the conventional rubber process either smoke-dried, usually at $60^{\circ} \mathrm{C}$ (Sheet Rubber) or dried by air $\left(\mathrm{Cr}^{\wedge}\right.$ epe Rubber) and then, the conventional rubber is pressed into big bales, where as the crumb rubbers are pressed into small bales (Bengtsson and Stenberg, 1996).

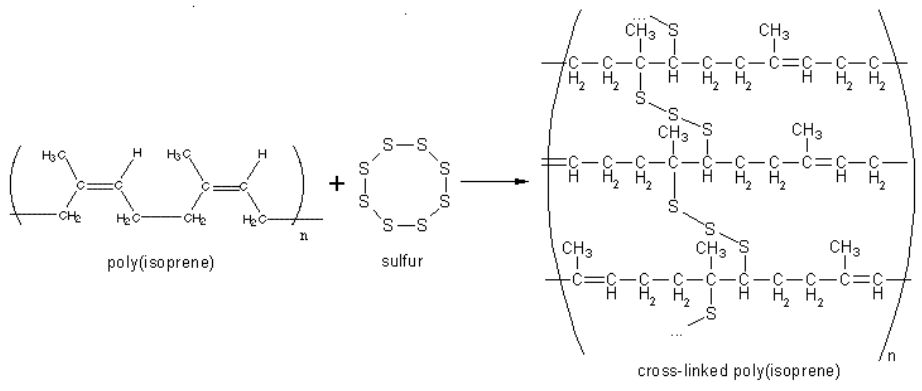

Figure 2: Chemical-rubber formula

Raw rubber becomes stiffer during storage. This is thought to be caused by chemical process in which rubber chains slowly become linked by reactions involving carbonyl groups (aldehyde) incorporated on the main rubber chain and amino acids present among the non-rubber constituents (Bengtsson and Stenberg, 1996). The process in above is called as 'storage hardening'.

\subsection{Vulcanization of Rubber.}

Raw rubber must be treated with additional chemical substances to improve the quality of the raw rubber before used to produce products. The process to mixing raw rubber with chemical substances is called as compounding processes. Generally, vulcanization refers to a specific of curing the rubber process that involving with high heat and curing agents. It is a chemical process in which the process occurs when the polymer molecules are linked to other polymer molecules by atomic bridges composed of atoms from the curing agents. This research to prediction heat transfer rubber with compares using partial differential equation.

The end product of the compounding process is a thin sheet of soft rubber compound that will be used as the 'blank' in the compression moulding process. The 'blank' contains curing agents needed to produce vulcanized 
rubber during the compression moulding process.

A rubber compound, before it is cured, may possess properties of a very viscous fluid, which has no mechanical stability and the times is 5 to 13 minutes, will flow and change its shape (Pongdhom and Thepsuwan, 2002). Therefore, the process of curing is vital in the production of rubber components, and mostly used in engineering industry and daily applications. Curing rubber components are harder, more durable and also more resistant to chemical attack. It is also making the surface of the material smoother and prevents it from sticking to metal or plastic chemical catalysts.

Curing is a term in polymer chemistry and engineering process that refers to the toughening or hardening of a polymer material by cross-linking polymer-chains, brought about by chemical additives, ultraviolet radiation, electron beam or heat. In rubber, the curing processes are also called as vulcanization.

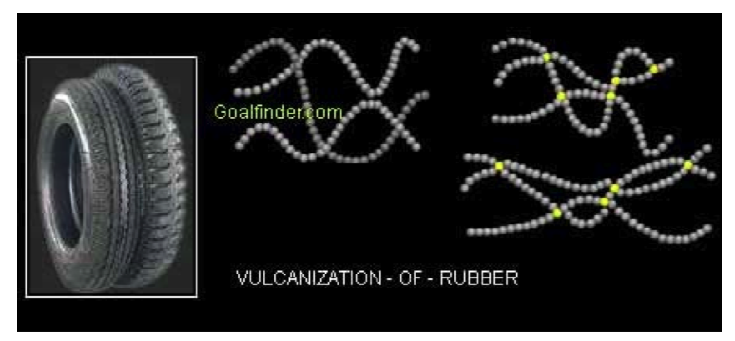

Figure 3: Vulcanization of rubber.

Vulcanization is generally considered to be an irreversible process, similar with other thermosets and must be contrasted strongly with thermoplastic processes (melt-freeze process), which characterize the behavior of the most modern polymers. The fundamental difference between rubbers and thermoplastics materials is that cured rubber compounds do not melt on heating. The chemical reaction takes place while the rubber is in the heated mould.

\section{Mathematical Modeling}

Mathematical modeling under consideration is the one-dimensional two-phase Stefan problem involving the appearance of a new phase. To predict the temperature of phase change is determined by:

$$
\rho_{l} C_{l} \frac{\partial T_{l}}{\partial t}=\lambda_{l} \frac{\partial^{2} T_{l}}{\partial x^{2}}+\rho_{l} \frac{\partial X}{\partial t}, 0<x<X(t)
$$

Due to this differential parameter, the behavior and treats simulation can also be derived by:

$$
\rho_{s} C_{s} \frac{\partial T_{s}}{\partial t}=\lambda_{s} \frac{\partial^{2} T_{s}}{\partial x^{2}}+\rho_{s} \frac{\partial X}{\partial t}, x>X(t)
$$

where $\rho, C$, and $\lambda$ represented as the density of rubber, heat capacity of rubber and thermal conductivity of rubber, respectively. The subscripts 1 and $\mathrm{s}$ in equation (1) and (2) indicate the liquid and solid state of the rubbers and $\mathrm{T}$ is the temperature $T(x, t)$. The contribution due to the conduction heat transfer and the exothermic reaction can be seen in the right-hand side of the equation. 
The initial condition is given by:

$$
T(x, 0)=T_{r}, T_{r}<T_{F}
$$

where $T_{r}$ is a known uniform temperature of the rubber compound before heating and $T_{F}$ are the fusion temperatures at the liquid-solid interface. Since two parallel planes bound the rubber compound, the boundary conditions of the rubber are found to be;

$$
T(0, t)=T(\alpha, t)=T(\beta, t)=T_{M}
$$

where $T_{M}$ is the temperature of the mould. Suppose there is a liquid-solid interface which separates the solid region from the liquid regions and this interface moves into the rubber when time elapses. Let $X_{1}(t)$ be the location of the interface in the rubber at time, $t$, therefore $X_{1}(t)$ can be determined by the internal energy equation,

$$
\rho_{l} L \frac{\partial X_{l}}{\partial t}=\lambda_{s} \frac{\partial T_{s}}{\partial x}+\lambda_{l} \frac{\partial T_{l}}{\partial t}
$$

where $\mathrm{L}$ is the latent heat for melting. This is a contribution with real applications.

\section{Numerical Methods}

There are some numerical methods to solve the partial differential equations in (1) and (2). The equations are reduced to a system of linear equations. Gauss-Seidel and Gauss-Seidel Red-Black methods are two common methods for solving, $A x=c$, that built by the German mathematicians Carl Friedrich Gauss and Philipp Ludwing von Seidel. The equation for the Gauss-Seidel method is: -

$$
\frac{T_{i, j+1}-T_{i, j}}{k}=\alpha_{n} \frac{T_{i+1, j}-2 T_{i, j}+T_{i-1, j}}{h^{2}},(\mathrm{i}=1,2,3, \ldots, \mathrm{n})
$$

The other Gauss-Seidel Red-Black method contains 2 sub-domains, $\alpha_{r}$ and $\alpha_{b}$. Moreover, there is communication between $\alpha_{r}$ and $\alpha_{b}$, and its solution is obtained through the following steps:

1. Grid calculation at $\alpha_{r}$,

$$
\frac{T_{i, j+1}-T_{i, j}}{k}=\alpha_{n} \frac{T_{i+1, j}-2 T_{i, j}+T_{i-1, j}}{h^{2}},(\mathrm{i}=1,3,5, \ldots, \mathrm{n}-1)
$$

2. Grid calculation at $\alpha_{b}$,

$$
\frac{T_{i, j+1}-T_{i, j}}{k}=\alpha_{n} \frac{T_{i+1, j}-2 T_{i, j}+T_{i-1, j}}{h^{2}},(\mathrm{i}=2,4,6, \ldots, \mathrm{n})
$$


For these researches, Gauss-Seidel Red-Black and Gauss-Seidel solutions are compared with the exact solution which is captured form Lembaga Getah Malaysia with parameters from equations (1) and (2).

\section{Numerical Result}

The following problem is solved using the Gauss-Seidel (GS) algorithm, with appropriate initial and boundary conditions, and it is compared with the Gauss-Seidel Red-Black (GSRB) algorithm. Figure 4 shows the temperature behavior, GS and GSRB, plotted against time, $t$ using the GS and GSRB algorithms. From the graph in Figure 4, GSRB algorithm is obviously better than GS algorithm.

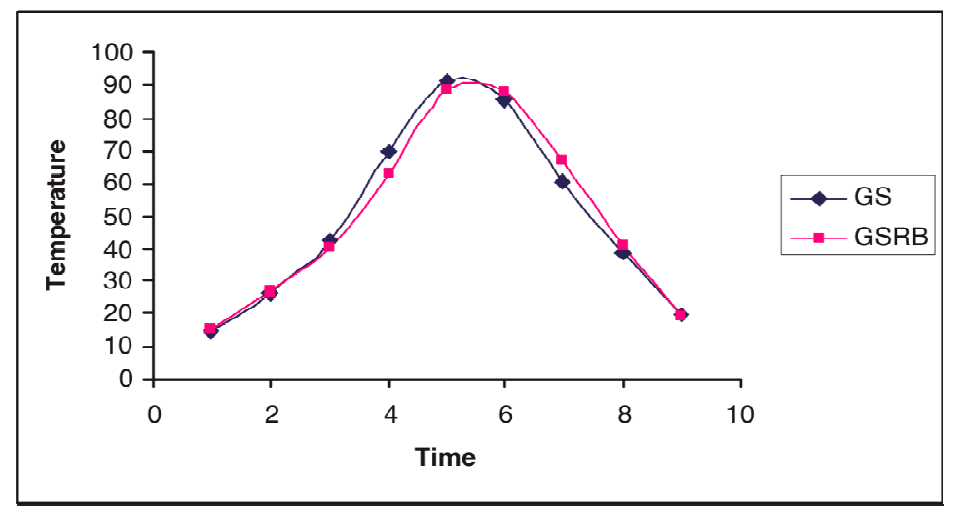

Figure 4. Temperature (T) vs time. Graph shows of temperature behavior using Gauss-Seidel (GS) and GaussSeidel Red-Black (GSRB) methods.

Table 1: Comparison of results between the exact, Gauss-Seidel and Gauss-Seidel Red-Black methods.

\begin{tabular}{|c|c|c|c|}
\hline Grid & Exact Solution & GS & GSRB \\
\hline 1 & 15 & 16.97 & 17.09 \\
\hline 2 & 26 & 25.23 & 26.24 \\
\hline 3 & 34 & 36.07 & 34.87 \\
\hline 4 & 65 & 67.45 & 60.46 \\
\hline 5 & 88 & 96.44 & 91.97 \\
\hline 6 & 85 & 88.05 & 90.86 \\
\hline 7 & 60 & 58.88 & 65.77 \\
\hline 8 & 40 & 36.3 & 37.57 \\
\hline 9 & 18 & 19.99 & 19.11 \\
\hline & & & \\
\hline
\end{tabular}

From the graph in Figure 5, we obtain the results for the time execution using GS algorithm required is $622 \mu \mathrm{s}$, while time execution if using GSRB algorithm required is $615 \mu \mathrm{s}$. The execution time for using GSRB algorithm is about 7 seconds faster than using sequential GS algorithm. This shows that sequential GSRB algorithm is significantly better than sequential GS algorithm. 


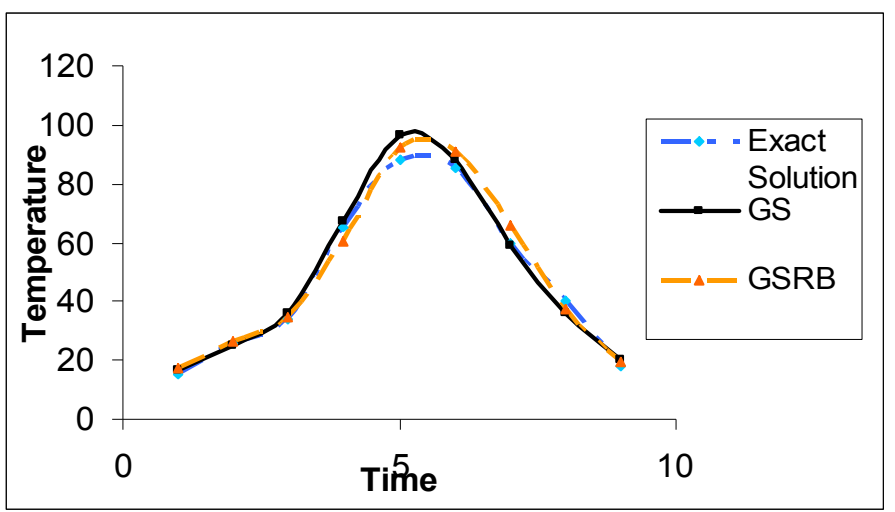

Figure 5: Temperature (T) vs time. Graph shows of temperature behavior between an exact result and the approximate result using Gauss-Seidel (GS) and Gauss-Seidel Red Black (GSRB) methods.

For both algorithms, the number of iterations for using GSRB algorithm is 117, while using GS algorithm is 112. This means the number of iterations in the GSRB algorithm is smaller than in the GS algorithm. Then, the accuracy or means square error for using GSRB method is smaller than GS method. Table 2 shows the numerical results through comparison using GSRB and GS methods and also proven that GSRB method is faster than the GS method.

Table 2: Numerical Analysis for Gauss-Seidel and Gauss-Seidel Red-Black methods

\begin{tabular}{|c|c|c|}
\hline Numerical Analysis & Gauss-Seidel & Gauss-Seidel Red-Black \\
\hline Time execution & $622 \mu \mathrm{s}$ & $615 \mu \mathrm{s}$ \\
\hline Number of Iteration & 10 & 10 \\
\hline Computational Complexity & 9 & 9 \\
\hline Accuracy & 3.22 & 2.66 \\
\hline Convergent & 0.05268 & 0.05266 \\
\hline
\end{tabular}

\section{Conclusion}

A mathematical model using the one dimensional parabolic equation involving phase change has been presented. The mathematical model of rubber curing is a combination of the transient heat conduction equation in the rubber compound and the rate equation of the chemical reaction. The mathematical models are providing more information than experimental. Some emphases are placed upon the stirring rate effect of the oil and coefficient of surface heat transfer.

The Gauss-Seidel Red-Black has been used for a method comparison. In addition, an iterative Gauss-Seidel method was studied to solve the parabolic equation. The results of the numerical analysis for the performance measurements such as time execution, number of iterations, computational complexity and accuracy indicate that GSRB is significantly better than the GS in terms of faster execution time.

Furthermore, the development of mathematical model in visualizing the temperature behavior can be easily extended into multi-dimensions parabolic equation. Upon successful achievement in our pilot tests, the mathematical model for the rubber curing will be extended into two- and three-dimensional approach.

The Gauss-Seidel Red-Black has been proven to be an effective iterative method and found to be well suited 
for parallel implementation on the distributed parallel computing system where data decomposition will run synchronously and concurrently at every time levels.

The end product of our research contributes in bringing benefits and advantages to the community of researchers from the rubber industry. Among these advantages are to help compression molders to optimize moulding conditions and part design. In addition, it will also reduce the time involved in the design of new

products and to propose technological conditions for the manufacturing process approaching optimal conditions, at reduced costs and material waste. It also offers tremendous flexibility to the researchers for determining the effects of different geometric features, different moulding and processing conditions and quality of the final product and can be used as a tool to maintain competitiveness of the rubber product in a globally aggressive market.

\section{Acknowledgement}

The authors acknowledge the Research Management Centre (RMC), Institute of Ibnu Sina, UTM and Ministry of Science, Technology and Environment Malaysia for the financial support using vot 79219.

\section{References}

[1] Ahmad Nazir Kamaruddin (2003). Application of VACAM-LABTM in tyre retreading (pre-cured tread).

[2] Azaar, K., El Brouzi, A., Granger, R. and Vergnaud, J. M. (1992). Modelling the cure of thermosetting polymers. E $\square$ ect of the coeffcient of surface heat transfer. Plastics and Rubber Processing and Applications. 18(1992). 95 - 102.

[3] Bengtsson, A. and Stenberg, B. (1996). Characterisation of Natural Rubber from Different Procuders. Progress in Rubber \& Plastic Technology. 12(1). 1 - 29.

[4] Broyer, E. (1976). Heat Transfer and Curing in Polymer Reaction Moulding. AIChE Journal. 22(2). 268 276.

[5] Caldwell, J. and Chiu, C. K. (1993). Numerical approaches to melting/solidification problems. In: Wrobel, L. C. and Brebbia, C. A. (1993). Computational Modelling of Free and Moving Boundary Problems II. Southampton: Computational Mechanics Publications. 6. Crank, J. (1984). Free and moving boundary problems. New York: Oxford University Press.

[6] Davis, B. A., Gramann, P. J., Osswald, T. A. and Rios, A. C. (2003). Compression Moulding. Munich: Hanser Publishers.

[7] Deqian, J. (1986). Calculation of heat transfer for the drag flow of rubber melt between two coaxial cylinders. Plastics and Rubber Processing and Applications. 6(1986). 291 - 295

[8] Eccles, C. R. H., Ellis, D. I., Heggs, P. J. and Wiktorowicz, R. (1990). Transient heat transfer model of blow moulding. Plastics and Rubber Processing and Applications. 14(1990). 219 - 222.

[9] Edwards, M. F., Georghiades, S. and Suvanaphen, P. K. (1981). A study of the cooling of blow moulded objects. Plastics and Rubber Processing and Applications. 1(1981). 161 - 165.

[10] Heggs, P. J., Houghton, J. M. and Ingham, D. B. (1995). Application of the enthalpy method to the blow moulding of polymers. Plastics and Rubber Processing and Applications. 23(1995). 203 - 210.

[11] Jaluria, Y. and Torrance, K. E. (2003). Computational Heat Transfer. 2nd Ed. New York: Taylor Francis.

[12] Jiji, L M. (2003). Heat Conduction. Mumbai: Jaico Publishing House.

[13] Mohammad Juma and Bafrnec, M. (2006). Heat Transfer Properties of Cord-reinforced Rubber Composites. Journal of Reinforced Plastics and Composites. 25(1). 1967 - 1975.

[14] Mohammad Juma and Bafrnec, M. (2004). Experimental Determination of Rubber Curing Reaction Heat Using the Transient Heat Conduction Equation. Chemical Papers. 58(1). 29 - 32.

[15] Halijah Osman, Norma Alias, Baharom Sanugi, The Parallelization of Charlie's Methods on Linear Heat Conduction Equation using Homogeneous Cluster Computing.12th Mathematics Science National Symposium, 23-24 Dec 2004, International Islamic University Malaysia. 